MATEC Web of Conferences 34, 04009 (2015)

DOI: $10.1051 /$ matecconf/ 20153404009

C Owned by the authors, published by EDP Sciences, 2015

\title{
A real-time non-contact monitoring method of subsea pipelines
}

\author{
Dalei Song ${ }^{1}$ Xueyan Ma ${ }^{1}$ Xinjian Fan ${ }^{1}$ Guojia Hou ${ }^{2}$ Jixing Sun ${ }^{3, a}$ Weiguo Shi ${ }^{3}$ Qingzhong Li ${ }^{1}$ \\ 1College of Engineering, Ocean University of China, Qingdao, 266100, China \\ 2College of Information Engineering, Qingdao University, Qingdao, 266071, China \\ ${ }^{3}$ CNOOC Changzhou Paint \& Coating Industry Research Institute Co.,Ltd, Tianjin, 300452, China
}

\begin{abstract}
To monitoring the subsea pipeline in real-time, a special potentiometric sensor array and a potential prediction model are presented in this paper. Firstly, to measure the potential of seawater, a special potentiometric sensor array with $\mathrm{Ag} / \mathrm{AgCl}$ all-solid-state reference electrodes is first developed in this paper. Secondly, according to the obtained distribution law of electric field intensities a prediction model of the pipeline potentials is developed. Finally, the potentiometric sensor array is applied in sink experiment and the prediction model is validated by the sink measurements. The maximum error for pipeline potential prediction model is $1.1 \mathrm{mV}$. The proposed non-contact monitoring method for subsea pipeline can predict the potential of sea pipeline in real-time, thus providing important information for further subsea pipeline maintenance.
\end{abstract}

\section{Introduction}

Marine Oil \& Gas resources is a major focus of China's energy, the exploitation of which brings wide usage of submarine pipelines. Integrity of subsea pipelines is a mandatory requirement in the modern offshore Oil \& Gas industry. A widely used cost-effective solution for subsea pipeline is CP [1-3] combined with external coating.

CP should be checked periodically, so that external corrosion is correctly prevented and proper protection is provided. In the offshore Oil \& Gas industry, the potential of pipeline is an important indicator of pipeline integrity.

Non-contact monitoring of offshore pipelines is usually carried out by a ROV (remotely operated vehicle) [4-6]. A tailored probe mounted on the ROV measures the seawater potentials in the surrounding of a subsea pipeline against a reference electrode embedded in the probe. But, this system cannot measure the potentials of pipeline directly. In order to overcome this disadvantage, we propose a special potentiometric sensor array which can measure seawater potential efficiently and a prediction model to monitoring pipeline potential real-time. The validity of the sensor array and the proposed prediction model is evaluated in the laboratory sink experiments.

\section{The Non-Contact Monitoring Method of Subsea Pipeline Under CP}

\subsection{The potentiometric sensor array}

A special potentiometric sensor array (Fig. 1) is first developed in this study. Twelve $\mathrm{Ag} / \mathrm{AgCl}$ all-solid-state reference electrodes are fixed on six vertical bars, and 
each reference electrode can measure the potential of its point. This way, we can obtain potential values of twelve different points simultaneously.

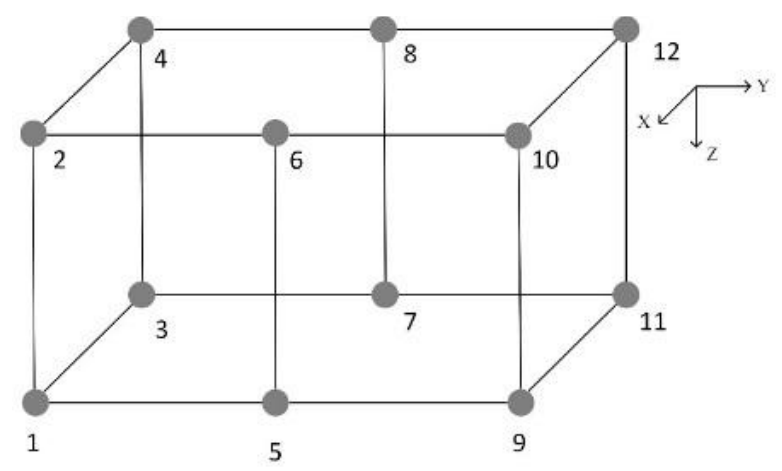

Figure 1 Potentiometric sensor array

The electric field intensity at point 5 has three components, namely $E_{x}, E_{y}$ and $E_{z}$ in $x, y, z$ direction respectively. Let the measured potential values of point 5 , point 6 , point 7 , and point 1 be $\varphi_{5}, \varphi_{6}, \varphi_{7}$ and $\varphi_{1}$, then the electric field intensity at point 5 can be calculated as follows.

$$
\begin{gathered}
E_{x}=\frac{\varphi_{7}-\varphi_{5}}{d_{x}} \\
E_{y}=\frac{\varphi_{1}-\varphi_{5}}{d_{y}} \\
E_{z}=\frac{\varphi_{6}-\varphi_{5}}{d_{z}}
\end{gathered}
$$

The electric field intensity $E_{5}$ of the 5 th reference electrode is

$$
E_{5}=\sqrt{E_{x}^{2}+E_{y}^{2}+E_{z}^{2}}
$$

Similarly, we can calculate the electric field intensity $E_{6}$ for the 6th reference electrode. So that we can obtain the electric field intensity of two points which in a line perpendicular to pipeline. With the potentiometric sensor array, we can measure the seawater potential around pipeline in three direction, so that we can obtain the electric field intensity.

\subsection{Distribution analysis of electric field intensity}

For the pipeline with one anode and one coating defect under $\mathrm{CP}$ in our laboratory sink, the seawater potentials around the pipeline are measured by the potentiometric sensor array and the electric field intensity value for each measuring point is calculated. Then the distribution law of electric field intensity is analysed at different position along the pipeline with respect to the radial distance between the measure point and the pipeline, where the measurement points are all above the pipeline.

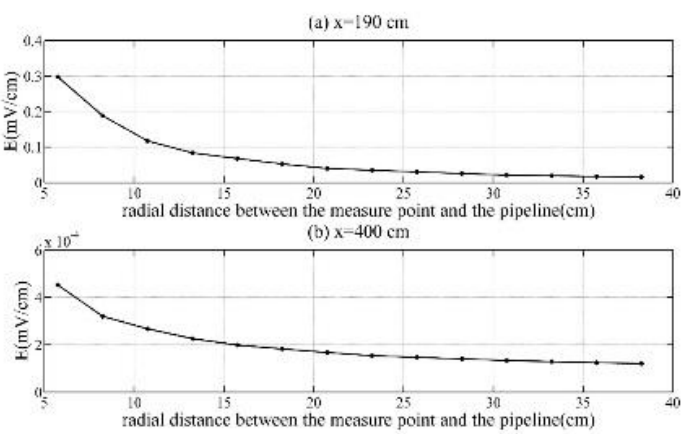

Figure 2 Electric field intensity distribution

Fig. 2 are two distribution curves of electric field intensity in two cross-sections $(x=190 \mathrm{~cm}$, and $x=400 \mathrm{~cm})$ along the pipeline respectively. It is seen that the distribution law of electric field intensity with respect to radial distance $d$ can be represented as an exponential function:

$$
E(x)=a \mathrm{e}^{-\lambda x},
$$

where $E$ is the electric field intensity of a spatial point, $x$ is the radial distance between the point and the pipeline, $a$ and $\lambda$ are two coefficients to be determined. The values of $E$ and $x$ at two points above the pipeline along the radial direction of the pipeline are calculated with the potentiometric sensor array, that is $\left(E_{1}, x_{1}\right)$ and $\left(E_{2}, x_{2}\right)$ are known. Then the two unknown coefficients of $a$ and $\lambda$ can be solved from equation (5) as:

$$
\left\{\begin{array}{c}
a=\frac{E_{2}{ }^{\frac{x_{1}}{x_{1}-x_{2}}}}{E_{1}^{\frac{x_{2}}{x_{1}-x_{2}}}} \\
\lambda=-\frac{\ln E_{1}-\ln E_{2}}{x_{1}-x_{2}}
\end{array}\right.
$$




\subsection{The prediction model of subsea pipeline potential}

For a uniform electric field, the electric field intensity $\mathrm{E}$ is calculated by

$$
E=\frac{U_{A B}}{x}
$$

where $U_{A B}$ is the potential difference between point $\mathrm{A}$ and point $\mathrm{B}$, and $x$ is the distance between $\mathrm{A}, \mathrm{B}$ along the direction of electric field intensity. The physical meaning of equation (7) is that the electric field intensity can be represented as the potential difference change per unit length along direction of $E$.

From equation (7), the potential $U_{0}$ of a point $P_{0}$ in a uniform electric field is calculated as

$$
U_{0}=U_{x}+E x
$$

where $U_{x}$ is a known potential value of a measure point $P_{x}$, and $x$ is the distance between $P_{0}$ and $P_{x}$ along the direction of $E$.

For a non-uniform electric field in seawater, the potential $U_{0}$ of a point $P_{0}$ can be computed by

$$
U_{0}=U_{x}+\int_{0}^{d} E(x) \mathrm{d} x
$$

where $d$ is the distance between $P_{0}$ and $P_{x}$ along the direction of $E$. It is noted that $E(x)$ is not a constant value in equation (9). Let $P_{0}$ be a point on surface of a pipeline, and $P_{x}$ be the measure point above the pipeline. Since the distance $d$ can be measured, if $E(x)$ is approximated with reasonable accuracy, then the potential value of the pipeline can be calculated with equation (9). Finally, the potential of the pipeline $U_{0}$ can be calculated based on the obtained $E(x)$ as

$$
\begin{aligned}
& U_{0}=U_{x}+\int_{0}^{d} E(x) \mathrm{d} x \\
= & U_{x}+\int_{0}^{d} \mathrm{a} \times e^{-\lambda x} \mathrm{~d} x \\
= & U_{x}-\frac{1}{\lambda a}\left(\mathrm{e}^{-\lambda d}-1\right),
\end{aligned}
$$

where $U_{x}$ is the potential value at point 5 , and $d$ is the radial distance between point 5 and the pipeline.

\section{Validation of the prediction model and the results}

For practical subsea pipelines of oil and gas in submarine environment, it is difficult to regulate the experimental factors such as length, position, size, and the damage degree of coating defects, burial depth of sea mud, and so on, so we use laboratory sink experiment to verify the effectiveness of the proposed potential perdition model. A pipeline under $\mathrm{CP}$ in a laboratory sink with one sacrifice anode and one coating defect was used to evaluate the proposed method.

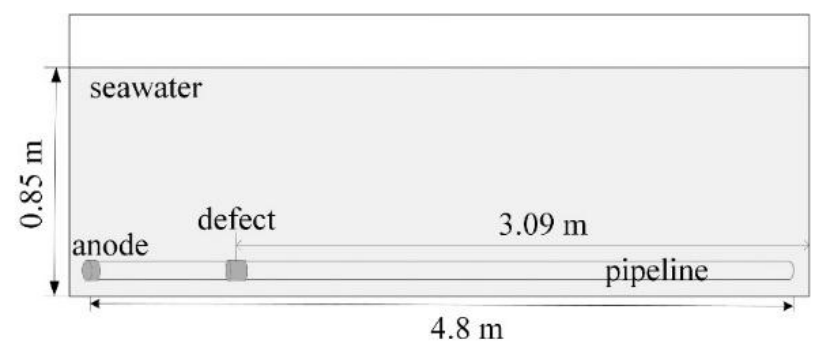

Figure 3 Schematic diagram of the experimental system

The laboratory sink is $5 \mathrm{~m}$ in length, $1 \mathrm{~m}$ in width, and $1 \mathrm{~m}$ in height (Fig. 3). The sink is filled with seawater, and the pipeline with one sacrifice anode and one coating defect under CP is fixed symmetrically $5 \mathrm{~cm}$ above the bottom of the sink. The detailed parameters of the experimental system are shown in Table 1.

Table 1 Parameters of the experimental system

\begin{tabular}{lll}
\hline \multicolumn{1}{c}{ pipeline } & \multicolumn{1}{c}{ seawater } & \multicolumn{1}{c}{ anode } \\
\hline $\operatorname{diameter}(\mathrm{mm}): 10$ & temperature $\left({ }^{\circ} \mathrm{C}\right): 2$ & $\operatorname{diameter}(\mathrm{mm}): 10$ \\
length $(\mathrm{m}): 4.8$ & $\operatorname{depth}(\mathrm{m}): 0.85$ & length $(\mathrm{mm}): 3.185$ \\
damage & $\operatorname{resistivity}(\Omega \cdot \mathrm{cm}):$ & $\operatorname{exposed}$ \\
$\operatorname{rate}(\%): 3.13$ & 96.8 & $\operatorname{area}\left(\mathrm{cm}^{2}\right): 1$ \\
\hline
\end{tabular}

The potentials and the corresponding threedimensional coordinates of the points in water space of the sink are first collected with the developed potentiometric sensor array and measuring system. 


\section{MATEC Web of Conferences}

Secondly, the radial distance between each point and the pipeline can be calculated. Then the each pair of measured potentials in the same radial direction and the corresponding distance is input to the proposed prediction model to compute the potential of the pipeline. Finally, the calculated potential values of the pipeline are compared with the actual measured potentials of the pipeline.
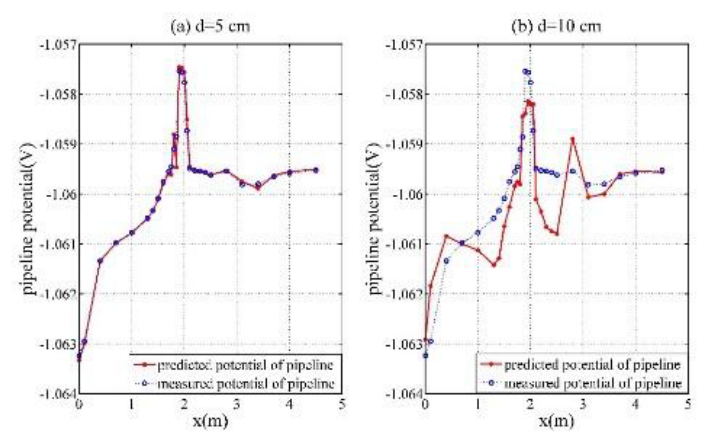

Figure 4 prediction result of the pipeline in the laboratory sink

Two typical prediction results are shown in Fig. 4. The maximum prediction error for the measured points with a radial distance of $5 \mathrm{~cm}$ to the pipeline is $0.3 \mathrm{mV}$, and that for the measured points with a radial distance of $10 \mathrm{~cm}$ to the pipeline is $1.1 \mathrm{mV}$. It is also noted that from Fig. 4 , at the positions of coating defects, the prediction model can follow large potential change of the pipeline with a smaller potential change of measured points as its input.

The calculated of the model is simply, so that we can realize the measure of the pipeline potential in real time. At the same time, the potential prediction model can follow the real pipeline potential accurately.

\section{Conclusions}

A real-time non-contact monitoring method of subsea pipelines is proposed to realize the monitoring of a subsea pipeline under CP. This method uses the measured potential above the pipeline measured by a potentiometric sensor array and its distance to the pipeline as the input to compute the potential of the pipeline.

Comparison to experimental result of a pipeline in a laboratory sink, the maximum prediction error is $1.1 \mathrm{mV}$, and for a pipeline with one coating defect, the prediction model can follow large potential change of the pipeline with a smaller potential change of measured points as its input.

The proposed potential prediction method for subsea pipeline can predict the potential of sea pipeline in real-time for non-contact detection systems, thus providing important information for further subsea pipeline maintenance.

\section{References}

1. P. Marcassoli, A. Bonetti, L. Lazzari, M. Ormellese, Mater. Corros. 2015, 66, 619.

2. P. Wang, Y. Huang, W. Xia, L. Luo, J. Lan, presented at ISOPE-2010, Beijing, China, June 20- June 25, 2010, pp. 211-217.

3. G. Hu, S. Zhang, B. Xiang, Chongqing Daxue Xuebao. 2008, 12, 1415

4. F. Shaffer, Ö. Sava, K. Lee, G. de Vera, Flow. Meas. Instrum. 2015, 43, 34.

5. G. De Tomi, F. Rocha, J.L. Castro, A.F. Silva, presented at OCEANS 2014, St. John's, NL, Canada, September 14 September 19, 2014.

6. E. Omerdic, D. Toal, G. Dooly, A. Kaknjo, presented at OCEANS 2014, St. John's, NL, Canada, September 14 September 19, 2014. 